\title{
AN Approach to REDUCE ENERGY Consumption in Cloud Data Centers Using HARMONY SEARCH ALGORITHM
}

\author{
Masoumeh Najafi ${ }^{1}$ and Keyvan Mohebbi ${ }^{\text {Corresponding Author, } 1,2}$ \\ ${ }^{1}$ Faculty of Computer Engineering, Najafabad Branch, Islamic Azad University, \\ Najafabad, Iran \\ ${ }^{2}$ Department of Electrical and Computer Engineering, Mobarakeh Branch, Islamic Azad \\ University, Mobarakeh, Isfahan, Iran
}

\begin{abstract}
Fast development of knowledge and communication has established a new computational style which is known as cloud computing. One of the main issues considered by the cloud infrastructure providers, is to minimize the costs and maximize the profitability. Energy management in the cloud data centers is very important to achieve such goal. Energy consumption can be reduced either by releasing idle nodes or by reducing the virtual machines migrations. To do the latter, one of the challenges is to select the placement approach of the migrated virtual machines on the appropriate node. In this paper, an approach to reduce the energy consumption in cloud data centers is proposed. This approach adapts harmony search algorithm to migrate the virtual machines. It performs the placement by sorting the nodes and virtual machines based on their priority in descending order. The priority is calculated based on the workload. The proposed approach is simulated. The evaluation results show the reduction in the virtual machine migrations, the increase of efficiency and the reduction of energy consumption.
\end{abstract}

\section{KEYWORDS}

Energy Consumption, Virtual Machine Placement, Harmony Search Algorithm, Server Consolidation, Binpacking Problem

\section{INTRODUCTION}

Cloud computing is a model based on computer networks which propose a new paradigm for the supply, use and delivery of services (including infrastructure, software, and platform) via the Internet. With the growth of the information technology, there is a need to perform computing tasks everywhere and every time. In addition, people want to conduct heavy computing tasks without owning expensive hardware and software. Cloud computing is the latest technology to answer these requirements. It provides a flexible infrastructure for a variety of computing and storage services, with the aid of virtual machines (VMs) [1,2].

From the perspective of a cloud provider, the important thing is to achieve maximum profitability by minimizing operating costs and guarantying the service level agreement (SLA). Therefore, energy management in cloud data centers has become very significant for achieving such objective. The rise of the cloud and the growing demand of its structure, has increased energy consumption dramatically in data centers. A typical data center with thousand racks requires ten megawatts of power which will be greater than the data center operating costs [2]. From 1990 to 
the present day energy consumption has doubled and is forecasted to increase by $2.2 \%$, almost every year, until 2040 [9]. This high energy consumption may increase the costs and reduce the profits for the cloud providers. As the temperature increases, reduction of equipment life, reduction of reliability, violations in QoS and SLA will be happened [6].

Due to the growing popularity of cloud computing users and increasing public awareness worldwide towards sustainable use of resources, researchers have devised a cloud with the implications of the environment friendly, namely green cloud computing to reduce both energy consumption and carbon dioxide emission. In this regard, several techniques are introduced. One of these is consolidation of VMs. In this technique, the workloads of multiple physical machines are placed on a single physical machine and the machine with low workload is off or hibernated. In order to consolidate, there are two challenges: 1- Choosing the best physical machine for placement of the VM on it so that we have the maximum physical resources and the least loss of resources. 2- If the mapping is not done correctly it will lead to an increase in the number of migrating VMs and because in the migration, CPU and bandwidth are used to transfer memory pages from the source to the destination node, therefore, it will lead to increase in energy.

The objective of this study is to choose the best physical machine to reallocate a VM and to reduce of migration of VMs in order to reduce energy consumption. To achieve this goal, the harmony search algorithm (HSA) will be adapted to select the appropriate physical machine for reallocation and the algorithm used in [34] will be used to reduce the number of migrations.

The remainder of this paper is as follows: In Section 2, the works related to the topic are explored. In Section 3, HSA will be described. In Section 4, our approach is proposed and evaluated. In Section 5, the simulation results will be analyzed. In Section 6, the conclusions and recommendations for future works will be discussed.

\section{RELATED WORKS}

Nathuji et al. [35] examined the problem of large-scale resource management in their virtual data centers and it was the first time that the power management technique was employed in the virtual systems. In addition, the hardware scalability and consolidation of VMs were used together and an energy management technique, namely the scalability of the software resources was applied by the authors. The aim of this approach was making use of guest VMs and the authors divided resource management in local and global politics. At the local level, the power management of the guest VM is in each physical machine and in global politics which is responsible for managing multiple VMs, in order to release the low load host and to save energy, VMs are consolidated. Results showed that the proposed approach leads to effective coordination of VMs and power management policies and energy consumption is reduced by $34 \%$.

Verma et al. [36] proposed a power-aware framework for a heterogeneous virtual environment. They used hardware techniques such as dynamic voltage and frequency scaling (DVFS) and virtualization to manage energy. A global manager is defined to allocate new VM and reallocate migrated VM. The migration cost is calculated using the size of VMs. The authors also compared several algorithms for solving the power optimization problem. They resolved some problems of the bin-packing approach, including variable size bin and the packaging cost by using the first-fit decreasing (FFD) algorithm. In FFD, first the bins are sorted in decreasing order, then starting from the biggest bin, they will be examined to fit inside a package. The results show that this framework has saved about $25 \%$ in power.

Reallocation of the VM using the power aware best fit decreasing (PABFD) algorithm on a set of heterogeneous physical machines to minimize power consumption in a virtualized data center was 
studied by Boyya and Belaglazov [2,3,4,5]. According to PABFD, first, VMs are sorted in decreasing order based on the efficiency of their processors. Then, the sorted machines will be allocated to the nodes that have the least power increase after allocation.

Fu and Zhou [13] studied the research works by the cloud team [3,5] to propose a new approach to reduce energy consumption. They used the policy of improving VM selection based on the CPU usage and allocating the migrated VM to a host using the minimum correlation coefficient (MCC) method. It means that by placing the migrated VM, the performance of its host will be degraded and the functionality of the existing VMs on this host will be disrupted.

Murtazaev and Oh [34] integrated the nodes using the VM migration algorithm in green cloud computing. Because migration costs a lot for the cloud supplier, the second goal of the authors was to minimize the number of migrations. Their approach outperforms the bin-packing heuristic algorithms, such as the first-fit decreasing algorithm.

Suresh kumar and Aramudhan [29] scheduled tasks using both harmony search and bat algorithms. The objective function that is considered in HSA, selects a solution and compares it with the worst solution available in the harmony memory. This approach will be used as a task scheduler service in the software as a service (SaaS) level.

Hoang et al. [18] offered a framework for real time Wireless Sensor Networks (WSNs) based on HSA and optimizes energy distribution in such networks by reducing the distance between their nodes. Harmony search optimizer algorithm executes in a reasonable time for real-time operations in order to improve the lifetime of WSNs and apply it in real world projects such as the environment temperature and fire detection. The results show that using the proposed protocol, the lifetime of WSNs is increased.

\section{HARMONY SEARCh ALgORITHM (HSA)}

The HSA was first introduced in [28]. It has been applied for many optimization problems, such as water distribution networks, modeling of underground water and energy saving. HSA is faster and more converged than the particle swam optimization (PSO) algorithm and genetics and has lower equations and parameters $[15,16]$.

In order to explain the HSA, the process of producing music is checked by a skilled musician. Harmony as the coordination in music is a name for complementary notes or complementary frequencies which are added to the main melody to transfer feelings and make the music more beautiful and more pleasant [28].

Consider the musicians of an orchestra. Each of them is a variable in HSA. The resulting harmony in the orchestra, in fact is an answer or solution vector and continuous exercises of musicians are the number of repetitions in HSA. As every harmony in the orchestra after production should be evaluated in terms of aesthetics, each solution in HSA should be evaluated with fitness function. In each iteration, musicians try to improve their new harmony, where the aesthetic of such harmony is better than the previous ones [28].

In order to maintain the best previous harmony in HSA, the harmony memory is used. This memory is implemented as a matrix where each row is a solution and the entries are the variables considered for each solution. The number of columns of the matrix shows the dimensions of the solution [28]. The number of rows in the matrix is called the harmony memory size (HMS). The last column of matrix is considered to save the fitness function of each row (solution). A view of 
the harmony memory matrix $(\mathrm{HM})$ is shown in Figure 1 , where $\mathrm{N}$ is the dimension of the solution.

$$
\mathrm{HM}=\left[\begin{array}{cccc:c}
x_{1}^{1} & x_{2}^{1} & \ldots & x_{N}^{1} & f\left(\mathbf{X}^{\mathbf{1}}\right) \\
x_{1}^{2} & x_{2}^{2} & \ldots & x_{N}^{2} & f\left(\mathbf{X}^{2}\right) \\
\vdots & \vdots & \ddots & \vdots & \vdots \\
x_{1}^{H M S} & x_{2}^{H M S} & \ldots & x_{N}^{H M S} & f\left(\mathbf{X}^{\mathrm{HMS}}\right)
\end{array}\right]
$$

Figure 1. Harmony Memory Matrix

The important thing in this algorithm is to find the best solution among existing solutions and to select the appropriate values for the parameters to increase the effectiveness and performance of this approach. The purpose of parameter settings is selecting the best values for parameters, so that the performance of the algorithm become optimal (best possible performance). These values may have a significant impact on the efficiency and effectiveness of the algorithm [16].

\section{The Proposed Approach for Reducing Energy Consumption in Cloud Data Centers}

This paper aims at energy efficiency in the infrastructure as a service (IaaS) level. The main technique used for improving the efficiency of resources in the data centers is virtualization. The proposed approach reduces both the VM migration and energy consumption, using dynamic placement of migrated VMs. This approach has four steps:

1- Sorting the hosts in descending order by their work loads

2- VMs are selected from the low load host for migration and are sorted in descending order by their ranking in the list of migration. It is worth noting that there is a possibility of migration if all VMs are able to migrate from the considered host. If this is impossible for a single VM, there is no migration from that host.

3- Placing the VMs from the list of migration to the target host considering the threshold of $70 \%$ (i.e., the VMs are given to the host that the ranking summation of the VM and the host is less than the threshold of $70 \%$ ).

4- Shutting down the low load host.

This research focuses on VM placement.VM placement can be considered as the bin packing problem, in that the bins are the hosts and packets are VMs. Because bin packing is a NP-hard problem [34], heuristic methods are used to solve them.

In order for consolidation and migration of VMs, the algorithm proposed in [34] is used and to calculate the rankings, the formulas introduced in [34] is applied. The optimal solution for the placement of migrated VMs is achieved using modified HSA. In this study, the fitness function is defined as follows: VM will be given to a host if the ranking summation of VM and the host is less than the threshold $(70 \%)$. The pseudo-code of the proposed approach to improve VM allocation to the host is illustrated in Figure 2. 


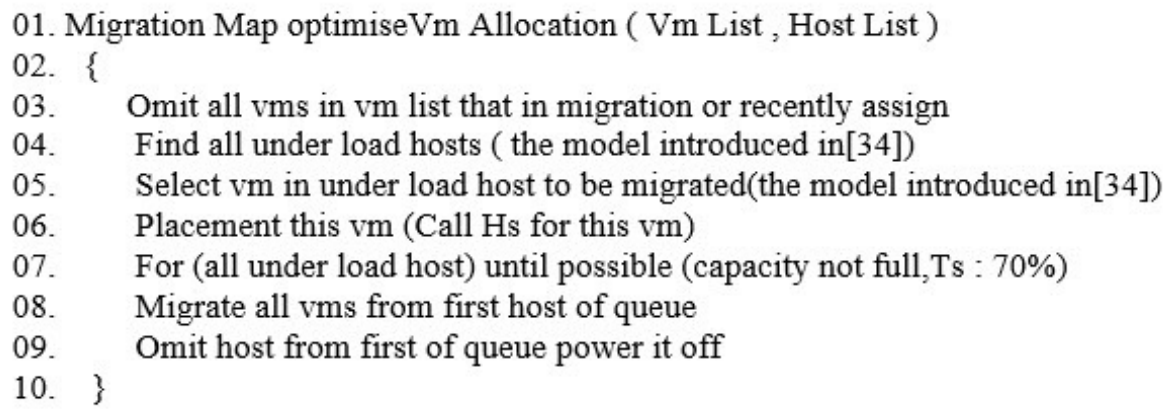

Figure 2. The Pseudo-code of the Proposed Approach

\section{SiMULATION RESUltS}

The CloudSim simulator is used to evaluate the proposed approach. The specification of the test environment used by the simulator is depicted in Table 1. The profile of the hosts is shown in Tables 2 and 3. The data set is obtained during ten business days of the entire test period [5], randomly from 800 host and the infrastructure layer (Table 4). For placing the migrated VM, to suitable host, HSA is implemented in the simulator. The algorithms and formulas proposed in [34] are applied.

The proposed approach is compared with PABFD with respect to the number of VM migration (Figure 3), the number of active hosts (Figure 4), energy consumption (Figure 5) and migration efficiency (Figure 6).

Table 1. Hardware and Software Test Environment

\begin{tabular}{|l|l|}
\hline CPU Model & INTEL \\
\hline Number of Cores & I7-2.0 GHz \\
\hline Memory & $6 \mathrm{~GB}$ \\
\hline Operating System & WIN8 \\
\hline Software & NETBEANS7.3 \\
\hline
\end{tabular}

Table 2. Profile of the Hosts

\begin{tabular}{|l|l|l|}
\hline \multicolumn{1}{|c|}{ Host } & \multicolumn{1}{c|}{ HP ProLiant G4 } & \multicolumn{1}{c|}{ HP ProLiant G5 } \\
\hline CPU & $1 \times$ Xeon 3040 $(1860 \mathrm{MHz})$ & $1 \mathrm{x}$ Xeon $3040(2660 \mathrm{MHz})$ \\
\hline Cores & 2 & 2 \\
\hline RAM (GB) & 80 & 80 \\
\hline Bandwidth (Gbits/s) & 10 & 10 \\
\hline
\end{tabular}


Table 3. Energy Consumption of the Hosts

\begin{tabular}{|l|l|l|}
\hline \multicolumn{1}{|c|}{ CPU Load } & \multicolumn{1}{c|}{ HP ProLiant G4 } & \multicolumn{1}{c|}{ HP ProLiant G5 } \\
\hline $0 \%$ & 86 & 93.7 \\
\hline $10 \%$ & 89.4 & 97 \\
\hline $20 \%$ & 92.6 & 101 \\
\hline $30 \%$ & 96 & 105 \\
\hline $40 \%$ & 99.5 & 110 \\
\hline $50 \%$ & 102 & 116 \\
\hline $60 \%$ & 106 & 121 \\
\hline $70 \%$ & 108 & 125 \\
\hline $80 \%$ & 112 & 129 \\
\hline $90 \%$ & 114 & 133 \\
\hline $100 \%$ & 117 & 135 \\
\hline
\end{tabular}

Table 4. Workload in the Simulator

\begin{tabular}{|l|l|}
\hline \multicolumn{1}{|c|}{ Workload Date } & \multicolumn{1}{c|}{ Number of VMs } \\
\hline $03 / 03 / 2011$ & 1052 \\
\hline $06 / 03 / 2011$ & 898 \\
\hline $09 / 03 / 2011$ & 1061 \\
\hline $22 / 03 / 2011$ & 1516 \\
\hline $25 / 03 / 2011$ & 1078 \\
\hline $03 / 04 / 2011$ & 1463 \\
\hline $09 / 04 / 2011$ & 1358 \\
\hline $11 / 04 / 2011$ & 1233 \\
\hline $12 / 04 / 2011$ & 1054 \\
\hline $20 / 04 / 2011$ & 1033 \\
\hline
\end{tabular}

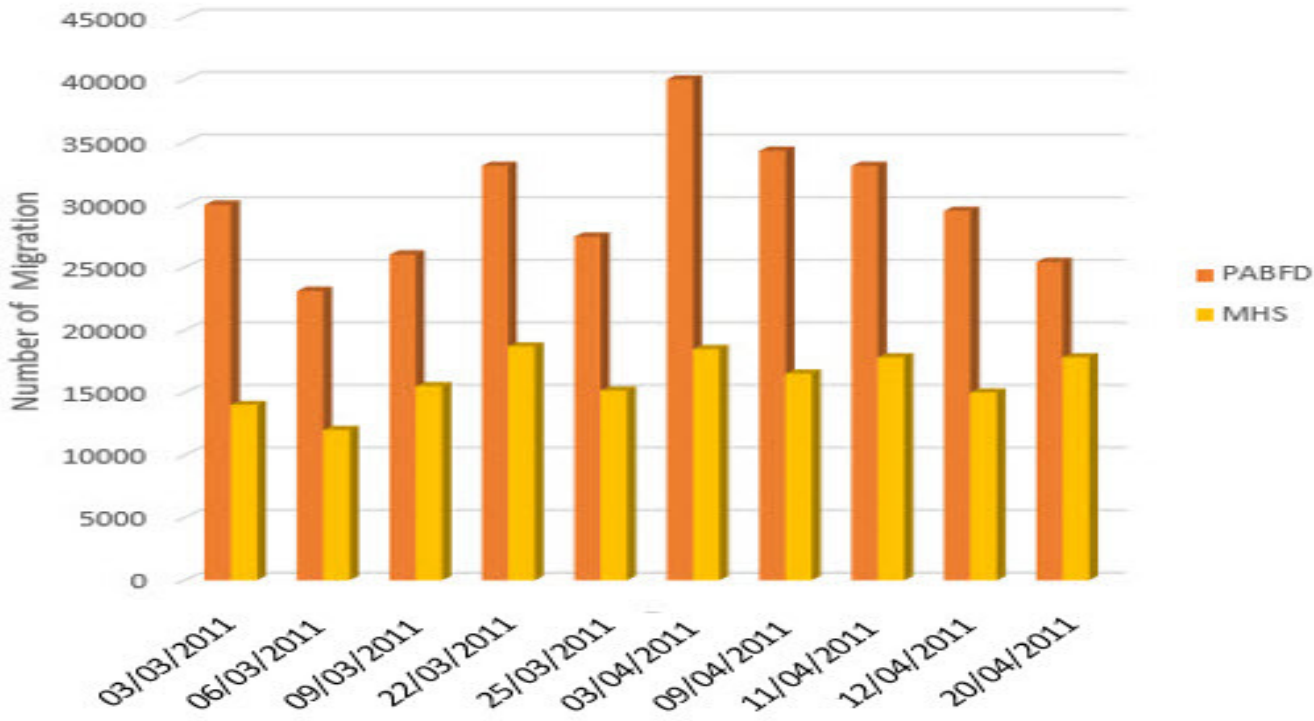

Figure 3. The Number of VMs Migrations 
International Journal on Cloud Computing: Services and Architecture (IJCCSA) Vol. 6, No. 4, August 2016

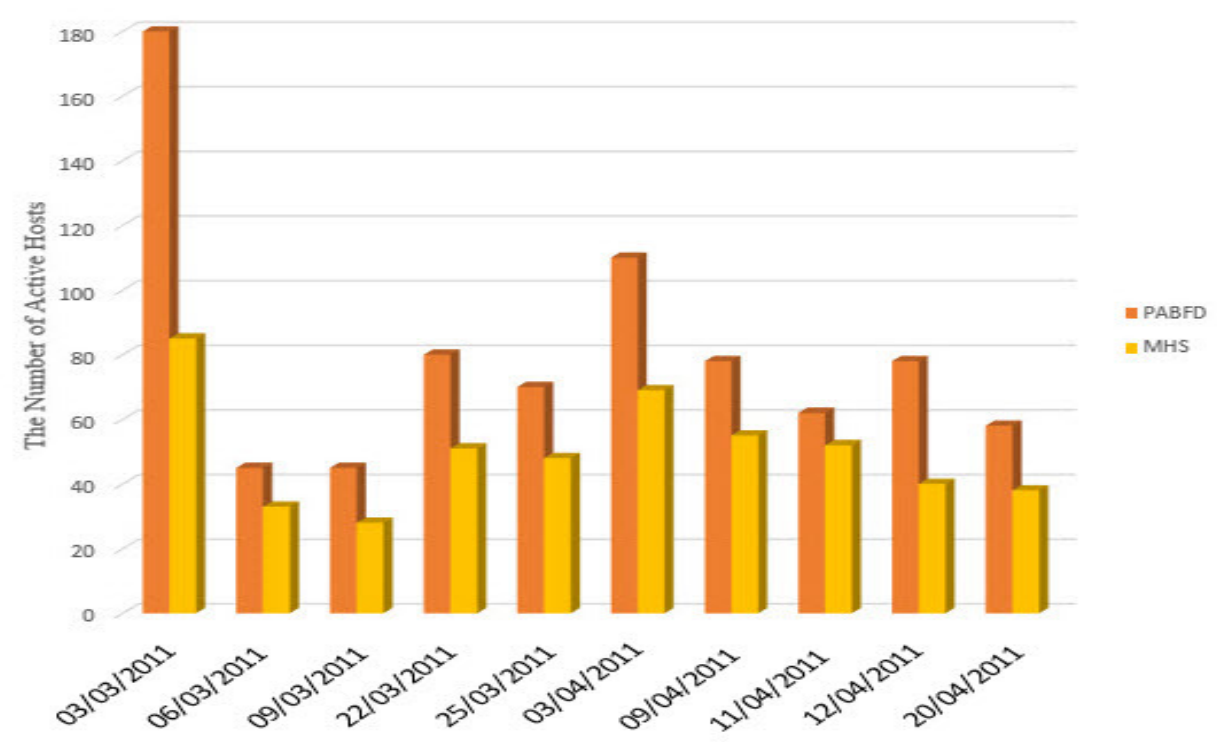

Figure 4. The Number of Active Nodes

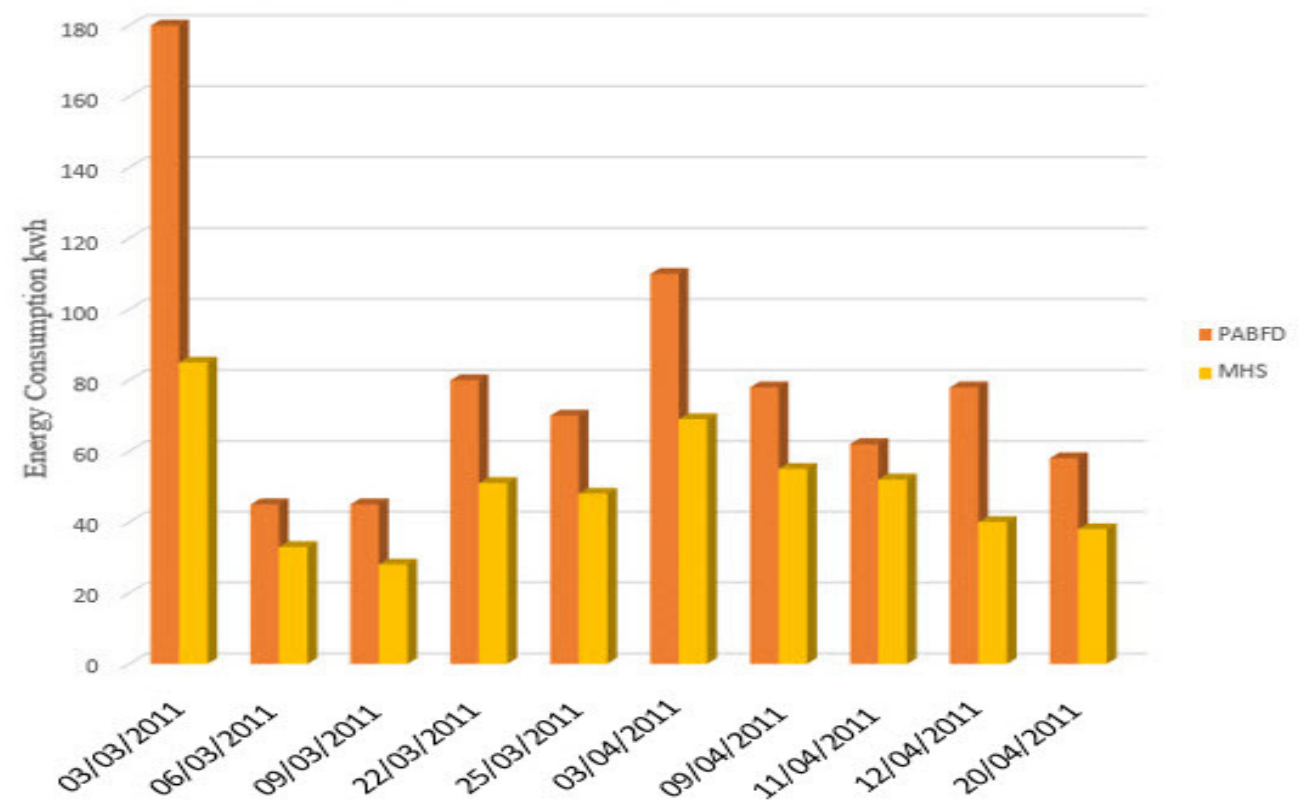

Figure 5. Energy Consumption in the Cloud Data Center 

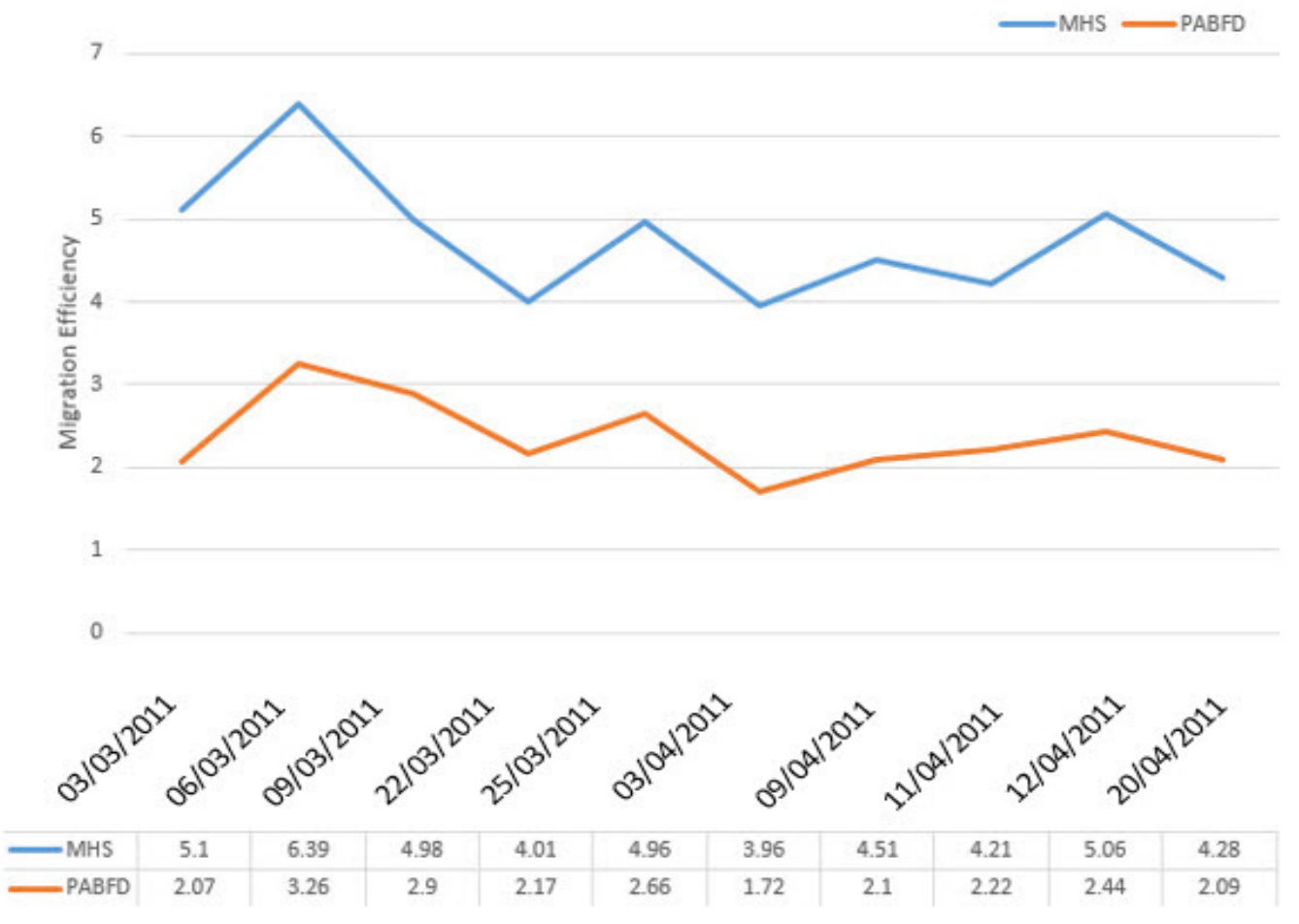

Figure 6. Immigration Efficiency

Simulation results show that the number of VMs migrations is reduced by $46 \%$, because according to the applied consolidation and migration models, the host with the lowest workload and the lowest number of VMs is selected for shutdown. In comparison with PABFD, the proposed approach reduces the numbers of active hosts by $40 \%$ and saves the energy consumption by $25 \%$.

\section{CONCLUSION AND FUtURE WORKS}

In this paper, an approach was proposed for energy efficiency in the cloud infrastructure layer. The low load hosts are detected and shut down and their VMs are migrated to the appropriate hosts. For the replacement, HSA is adapted. For the evaluation, the CloudSim simulator was used. The results of the comparative evaluation shows that the proposed approach outperforms PABFD with respect to the number of migrations, the number of active hosts, energy efficiency and migration efficiency parameters.

HSA has simple structure and may be combined with other meta-heuristics, in order to solve the problem of this study. For example, ant colony algorithm can be used to initialize the harmony memory, or HSA may be combined with the PSO algorithm to reduce energy consumption. In order to minimize response times in the cloud, the parallelized version of HSA can be used. 


\section{REFERENCES}

[1] A.Atrey, N. Jain ,Iyengar ,"A Study on Green Cloud Computing" International Journal of Grid and Distributed Computing,vol. 6,p. 93-102, (2013).

[2] A.Beloglazov, J. Abawajy , R. Buyya ,"Energy-aware resource allocation heuristics for efficient management of data centers for Cloud computing" vol. 28,p.755-768, (2012).

[3] A.Beloglazov, R. Buyya ,"Adaptive Threshold-Based Approach for Energy Efficient Consolidation of Virtual Machines in Cloud Data Centers" ,p.1-6, (2010).

[4] A.Beloglazov, R. Buyya ,"Energy Efficient Resource Management in Virtualized Cloud Data Centers" ,p.826-831, (2010).

[5] A.Beloglazov, R. Buyya ,"Optimal Online Deterministic Algorithms and Adaptive Heuristics for Energy and Performance Efficient Dynamic Consolidation of Virtual Machines in Cloud Data Center",vol. 24,p.1397-1420, (2012).

[6] A.Beloglazov, R. Buyya, Y. C. Lee , A. Zomaya ,"A Taxonomy and Survey of Energy-Efficient Data Centers and Cloud Computing Systems" ,vol. 82,p.1-50, (2011).

[7] R.Buyya, A. Beloglazov,"OpenStack Neat: a framework for dynamic and energy-efficient consolidation of virtual machines in OpenStack clouds" vol.27(5),p.1310-1333, (2015).

[8] R.Buyya, A. Beloglazov , J. Abawajy ,"Energy-efficient management of data center resources for Cloud computing" in Proceedings of the International Conference on Parallel and Distributed Processing Techniques and Applications (PDPTA),p. 1-12, (2010).

[9] P.company, www.gartner.com, (2015).

[10] S.E.Dashti, A. M. Rahmani ,"Dynamic VMs placement for energy efficiency by PSO in cloud computing", Experimental \& Theoretical Artificial,Intelligence,p.1-16, (2015).

[11] H.Duan, Q. Luo, Y. Shi , G. Ma ,"Hybrid Particle Swarm Optimization and Genetic Algorithm for Multi-UAV Formation Reconfiguration" , IEEE Computational Intelligence Society,vol. 8(3),p.16 27, (2013).

[12] M.Eissa, T. Abdel-hameed,"A Novel Approach for Optimum Number and Location of FACTS Devices on IEEE-30 Bus Grid using MetaHeuristicbasd Harmony Search",p. 1 - 10, (2013).

[13] X.FU, C. ZHOU ,"Virtual machine selection and placement for dynamic consolidation in Cloud computing environment" ,vol. 9(2),p.322-330, (2015).

[14] Y.Gaoa, H. Guana, Z. Qia, Y. Houb ,L. Liu ,"A multi-objective ant colony system algorithm for virtual machine placement in cloud computing", Journal of Computer and System Sciences ,vol.79(8),p.1230-1242, (2013).

[15] Z.W.Geem, J. H. Kim , G. V. Loganathan ,"A New Heuristic Optimization Algorithm: Harmony Search" ,vol. 76,p. 60-68, (2001).

[16] E.Khorram, M. Jaberipour ,"Harmony search algorithm for solving combined heat and power economic dispatch problems" ,Energy Conversion and Management ,vol.52(2),p. 1550-1554, (2011).

[17] A.Khosravi, S. K. Garg, R. Buyya ,"Energy and Carbon-Efficient Placement of Virtual Machines in Distributed Cloud Data Centers" ,p.317-328, (2013)

[18] D.C.Hoang, P. Yadav, R. Kumar, S. K. Panda ,"Real-Time Implementation of a Harmony Search Algorithm-Based Clustering Protocol for Energy-Efficient Wireless Sensor Networks" ,IEEE Computer Society ,vol.10(1),p.774 - 783, (2014).

[19] M.R.V.Kumar, S. Raghunathan ,"Heterogeneity and thermal aware adaptive heuristics for energy efficient consolidation of virtual machines in Infrastructure clouds", Journal of Computer and System Sciences ,vol.82(2),p. 1-30, (2015).

[20] T.Mastelic, A. Oleksiak, H. Claussen, I. Brandic , J.-M. Pierson ,"Cloud Computing: Survey on Energy Efficiency" Journal ACM Computing Surveys (CSUR),vol. 47(2),p. 33, (2015).

[21] K.Maurya, R. Sinha ,"Energy Conscious Dynamic Provisioning of Virtual Machines using Adaptive Migiration Thresholds in Cloud Data Center",p. 74-82, (2013).

[22] K.maurya, R. sinha ,"Energy Conscious Dynamic Provisioning Of Virtual Machines Using Adaptive Migration Thresholds in Cloud Data center ",vol. 2(3),p. 74-82, (2013).

[23] N.Quang-Hung, P. D. Nien, N. H. Nam, N. H. Tuong, N. Thoai ,"A Genetic Algorithm for PowerAware Virtual Machine Allocation in Private Cloud", Springer Berlin Heidelberg ,vol.7804, p. 183$191,(2013)$ 
[24] S.R.Suraj,R.Natchadalingam ,"Adaptive Genetic Algorithm for Efficient Resource Management in Cloud Computing" ,International Journal of Emerging Technology and Advanced Engineering , vol.4(2), p.350-356,(2014).

[25] J.Sekhar, G. Jeba ,"Energy Efficient VM Live Migration in Cloud Data Centers." IJCSN International Journal of Computer Science and Network, vol.2(2),p. 71-75,(2013).

[26] S.Tuo, J. Zhang, L. Yong, X. Yuan, B. Liu , X. Xub,F. a. Dengb, "A harmony search algorithm for high-dimensional multimodal optimization problems", Digital Signal Processing, vol.46, p.151$163,(2015)$

[27] Sh.Wang, Z. Liu, Z. Zheng, Q. Sun , F. Yang , "Particle Swarm Optimization for EnergyAwareVirtual Machine Placement Optimization in Virtualized Data Centers", 19th IEEE International Conference on Parallel and Distributed Systems,p. 102 - 109, (2013).

[28] X-S.Yang, "Harmony Search as a Metaheuristic Algorithm", Springer Berlin, vol.191, p.1-14,(2009)

[29] V.Suresh Kumar,M. Aramudhan" Trust Based Resource Selection in Cloud Computing Using Hybrid Algorithm”,I.J. Intelligent Systems and Applications, 2015, p.59-64 .Published Online July 2015 in MECS DOI: 10.5815/ijisa.2015.08.08

[30] Yongqiang Gao, Haibing Guan ,Zhengwei Qi, Yang Hou, Liang Liu,” A multi-objective ant colony system algorithm for virtual machine placement in cloud computing", 2013,http://dx.doi.org/10.1016/j.jcss.2013.02.004,p.1-13

[31] AkhilGoyal,Navdeep S. Chahal," A Proposed Approach for Efficient Energy Utilization in Cloud Data Center",International Journal of Computer Applications (0975 - 8887) Volume 115 - No. 11, April 2015,p.24-27

[32] ElinaPacini,Cristian Mateos, Carlos Garc'1aGarino,’Dynamic Scheduling based on Particle Swarm Optimization for Cloud-based Scientific Experiments",CLEI ELECTRONIC JOURNAL, VOLUME 14, NUMBER 1, PAPER 2, APRIL 2014.

[33] Gao, Y., Guan, H., Qi, Z., Hou, Y., \& Liu, L. (2013). A multi-objective ant colony system algorithm for virtual machine placement in cloud computing. Journal of Computer and System Sciences, 79, 1230-1242. doi:10.1016/j.jcss.2013.02.004

[34] A. Murtazaev,S.Oh,"Sercon: Server Consolidation Algorithm using Live Migration of Virtual Machines for Green Computing " ,IETE TECHNICAL REVIEW ,vol. 28, p.1-20, (2012)

[35] R.Nathuji, K. Schwan ,"VirtualPower: Coordinated power management in virtualized enterprise systems" ,ACM SIGOPS Operating Systems Review,vol. 41,p. 265-278, (2007).

[36] A.Verma, P. Ahuja ,A. Neogi, "pMapper: power and migration cost aware application placement in virtualized systems", n Proceedings of the 9th ACM/IFIP/USENIX International Conference on Middleware, p. 243-264,(2008). 